\title{
Existence of Mild Solutions and Controllability of Fractional Impulsive Integrodifferential Systems with Nonlocal Conditions
}

\author{
Haiyong Qin, ${ }^{1,2}$ Zhenyun $\mathrm{Gu}^{2}{ }^{2}$ Youliang $\mathrm{Fu}^{1}$ and Tongxing $\mathrm{Li}^{3,4}$ \\ ${ }^{1}$ School of Control Science and Engineering, Shandong University, Jinan, Shandong 250061, China \\ ${ }^{2}$ School of Mathematics, Qilu Normal University, Jinan, Shandong 250013, China \\ ${ }^{3}$ LinDa Institute of Shandong Provincial Key Laboratory of Network Based Intelligent Computing, Linyi University, Linyi, \\ Shandong 276005, China \\ ${ }^{4}$ School of Information Science and Engineering, Linyi University, Linyi, Shandong 276005, China
}

Correspondence should be addressed to Tongxing Li; litongx2007@163.com

Received 11 May 2017; Accepted 24 July 2017; Published 20 September 2017

Academic Editor: Xinguang Zhang

Copyright (C) 2017 Haiyong Qin et al. This is an open access article distributed under the Creative Commons Attribution License, which permits unrestricted use, distribution, and reproduction in any medium, provided the original work is properly cited.

\begin{abstract}
This paper is concerned with the existence results of nonlocal problems for a class of fractional impulsive integrodifferential equations in Banach spaces. We define a piecewise continuous control function to obtain the results on controllability of the corresponding fractional impulsive integrodifferential control systems. The results are obtained by means of fixed point methods. An example to illustrate the applications of our main results is given.
\end{abstract}

\section{Introduction}

In recent decades, existence of mild solutions of nonlocal Cauchy problems has been investigated extensively by many researchers (see [1-15] and the references cited therein). The study of abstract nonlocal semilinear initial value problems was initiated by Byszewski and Lakshmikantham [11] and Byszewski [12]. Byszewski [12] considered the existence and uniqueness of mild, strong, and classical solutions of nonlocal Cauchy problems. Lin and Liu [8] studied the existence and uniqueness of mild and classical solutions of semilinear integrodifferential equations with nonlocal Cauchy problems. Using Krasnoselskii's fixed point theorem, Schauder's fixed point theorem, and Banach contraction principle, Zhou and Jiao [13] obtained several criteria on the existence and uniqueness of mild solutions of nonlocal Cauchy problems for fractional evolution equations without impulse.

Such analysis on nonlocal Cauchy problems is important from an applied viewpoint, since the nonlocal condition has a better effect in applications than a classical initial one. For instance, the diffusion phenomenon of a small amount of gas in a transparent tube can be given a better description than using the usual local Cauchy problem. On the other hand, controllability of nonlocal problems in Banach spaces has become an active area of investigation; we refer the reader to, for example, the papers [16-29]. The most common method is to transform the controllability problem into a fixedpoint problem of solutions for an appropriate operator in a function space, that is, the existence problem of differential and integrodifferential equations. Unfortunately, by [16], we know that the concept of mild solutions used in $[14,15,17]$ was not suitable for fractional evolution systems.

Chang et al. [18] investigated the controllability of a class of first-order semilinear differential systems with nonlocal initial conditions in a Banach space:

$$
\begin{aligned}
& x^{\prime}(t)=A x(t)+f(t, x(t))+B u(t), \\
& t \in J=[0, b],
\end{aligned}
$$

$$
x(0)+g(x)=x_{0} \in \mathbb{X},
$$

where $A$ generates a strongly continuous, not necessarily compact, semigroup $(T(t))_{t \geq 0}$ in the Banach space $\mathbb{X}$. Sufficient conditions for the controllability of the first-order 
semilinear differential system with nonlocal initial conditions were established. The approach used is Sadovskii's fixed point theorem.

Balachandran et al. [19] discussed the controllability of a class of fractional integrodifferential systems with nonlocal conditions in a Banach space:

$$
\begin{array}{r}
\frac{d^{q} x(t)}{d t^{q}}=A x(t)+f(t, x(t),(H x)(t))+B u(t), \\
t \in J=[0, b],
\end{array}
$$

$$
x(0)+g(x)=x_{0} \in \mathbb{X} .
$$

Motivated by the work of the above papers and wide applications of nonlocal Cauchy problems in various fields of natural sciences and engineering, in this paper, we study the existence of nonlocal problems for a class of fractional impulsive integrodifferential systems in a Banach space of the following type:

$$
\begin{gathered}
D_{t}^{q} x(t)=A x(t)+f(t, x(t),(H x)(t)), \\
t \in I=[0, b], t \neq t_{k}, \\
\left.\Delta x\right|_{t=t_{k}}=I_{k}\left(x\left(t_{k}^{-}\right)\right), \quad k=1,2, \ldots, m, \\
x(0)+g(x)=x_{0} \in \mathbb{X},
\end{gathered}
$$

where $(H x)(t)=\int_{0}^{t} h(t, s, x(s)) d s$ and $D_{t}^{q}$ is the Caputo fractional derivative $(0<q<1)$; the state $x(\cdot)$ takes values in the Banach space $\mathbb{X} . A: D(A) \subseteq \mathbb{X} \rightarrow \mathbb{X}$ is the infinitesimal generator of a strongly continuous semigroup $(T(t))_{t>0}$ of uniformly bounded operators in $\mathbb{X}$, and $A$ is a bounded linear operator. $f: I \times \mathbb{X} \times \mathbb{X} \rightarrow \mathbb{X}$ is a given $\mathbb{X}$-value function; $h: \Delta \times \mathbb{X} \rightarrow \mathbb{X}$ is continuous; here $\Delta=\{(t, s): 0 \leq s \leq t \leq b\}$, $I_{k}: \mathbb{X} \rightarrow \mathbb{X}, 0=t_{0}<t_{1}<\cdots<t_{m}<t_{m+1}=b$, $\left.\Delta x\right|_{t=t_{k}}=x\left(t_{k}^{+}\right)-x\left(t_{k}^{-}\right), x\left(t_{k}^{+}\right)=\lim _{h \rightarrow 0^{+}} x\left(t_{k}+h\right)$, and $x\left(t_{k}^{-}\right)=$ $\lim _{h \rightarrow 0^{-}} x\left(t_{k}+h\right)$ represent the right and left limits of $x(t)$ at $t=t_{k}$, respectively. Using the similar method and a piecewise continuous control function, we consider the controllability of a class of fractional impulsive integrodifferential systems with nonlocal initial conditions:

$$
\begin{aligned}
D_{t}^{q} x(t) & =A x(t)+f(t, x(t),(H x)(t))+B u(t), \\
t & \in I=[0, b], t \neq t_{k}, \\
\left.\Delta x\right|_{t=t_{k}} & =I_{k}\left(x\left(t_{k}^{-}\right)\right), \quad k=1,2, \ldots, m, \\
x(0)+g(x) & =x_{0} \in \mathbb{X},
\end{aligned}
$$

where $B$ is a bounded linear operator from $U$ to $\mathbb{X}$ and the control function $u(\cdot)$ is given in $L^{2}[I, U]$, with $U$ as a Banach space.

We study the nonlocal initial problem (3) that describes a more general form than the previous ones reported in $[18,19]$. We introduce a suitable concept of PC-mild solutions for nonlocal initial problem (3). We not only study the existence and uniqueness of a mild solution for impulsive fractional semilinear integrodifferential equation (3) but also define a piecewise continuous control function and present the results on the controllability of the corresponding fractional impulsive integrodifferential system (4) which include some known results obtained in $[14,17]$. Assumptions in our results are less restrictive.

\section{Preliminaries and Lemmas}

Throughout this paper, let us consider the set of functions $\mathrm{PC}[I, \mathbb{X}]=\left\{x: I \rightarrow \mathbb{X} \mid x \in C\left[\left(t_{k}, t_{k+1}\right), \mathbb{X}\right]\right.$ and there exist $x\left(t_{k}^{-}\right)$and $x\left(t_{k}^{+}\right), k=0,1,2, \ldots, m$, with $\left.x\left(t_{k}^{-}\right)=x\left(t_{k}\right)\right\}$. Endowed with the norm $\|x\|_{\mathrm{PC}}=\sup _{t \in I}\|x(t)\|$, it is easy to verify that $\left(\mathrm{PC}[I, \mathbb{X}],\|\cdot\|_{\mathrm{PC}}\right)$ is a Banach space. Let $L_{B}(\mathbb{X})$ be the Banach space of all linear and bounded operators on $\mathbb{X}$. For a $C_{0}$-semigroup $(T(t))_{t \geq 0}$, we set $M_{1}=\sup _{t \in I}\|T(t)\|_{L_{B}(\mathbb{X})}$. For each positive constant $r$, we set $B_{r}=\{x \in \operatorname{PC}[I, \mathbb{X}]$ : $\|x\| \leq r\}$. Obviously, $B_{r}$ is a bounded closed and convex subset.

Definition 1. The fractional integral of order $\gamma$ with the lower limit zero for a function $f$ is defined as

$$
I^{\gamma} f(t)=\frac{1}{\Gamma(\gamma)} \int_{0}^{t} \frac{f(s)}{(t-s)^{1-\gamma}} d s, \quad t>0, \quad \gamma>0,
$$

provided that the right side is point-wise defined on $[0, \infty)$, where $\Gamma(\cdot)$ is the gamma function.

Definition 2. The Riemann-Liouville derivative of order $\gamma$ with the lower limit zero for a function $f:[0, \infty) \rightarrow \mathbb{R}$ can be written as

$$
\begin{aligned}
{ }^{L} D^{\gamma} f(t)=\frac{1}{\Gamma(n-\gamma)} \frac{d^{n}}{d t^{n}} \int_{0}^{t} \frac{f(s)}{(t-s)^{1-n+\gamma}} d s, \\
\quad t>0, n-1<\gamma<n .
\end{aligned}
$$

Definition 3. The Caputo derivative of order $\gamma$ for a function $f:[0, \infty) \rightarrow \mathbb{R}$ can be written as

$$
\begin{aligned}
D_{t}^{\gamma} f(t)={ }^{L} D^{\gamma}\left(f(t)-\sum_{k=0}^{n-1} \frac{t^{k}}{k !} f^{(k)}(0)\right) & \\
& t>0, n-1<\gamma<n .
\end{aligned}
$$

Remark 4. If $f$ is an abstract function with values in $\mathbb{X}$, then integrals that appear in Definitions 1-3 are taken in Bochner's sense.

Definition 5 (see [20]). Let $\mathbb{X}$ be a Banach space; a oneparameter family $T(t), 0 \leq t<\infty$, of bounded linear operators from $\mathbb{X}$ to $\mathbb{X}$ is a semigroup of bounded linear operators on $\mathbb{X}$ if

(1) $T(0)=I$; $I$ is the identity operator on $\mathbb{X}$;

(2) $T(t+s)=T(t) T(s)$ for every $t, s \geq 0$ (the semigroup property).

A semigroup of bounded linear operators, $T(t)$, is uniformly continuous if $\lim _{t \downarrow 0}\|T(t)-I\|=0$.

Definition 6 (see [21]). By a PC-mild solution of system (3), we mean a function $x \in \mathrm{PC}[I, \mathbb{X}]$ that satisfies the following integral equation: 


$$
x(t)=\left\{\begin{array}{l}
\mathscr{T}(t)\left[x_{0}-g(x)\right]+\int_{0}^{t}(t-s)^{q-1} \mathcal{S}(t-s) f(s, x(s),(H x)(s)) d s, \quad t \in\left[0, t_{1}\right], \\
\mathscr{T}(t)\left[x_{0}-g(x)\right]+\int_{0}^{t}(t-s)^{q-1} \mathcal{S}(t-s) f(s, x(s),(H x)(s)) d s+\mathscr{T}\left(t-t_{1}\right) I_{1}\left(x\left(t_{1}^{-}\right)\right), \quad t \in\left(t_{1}, t_{2}\right], \\
\vdots \\
\mathscr{T}(t)\left[x_{0}-g(x)\right]+\int_{0}^{t}(t-s)^{q-1} \mathcal{S}(t-s) f(s, x(s),(H x)(s)) d s+\sum_{k=1}^{m} \mathscr{T}\left(t-t_{k}\right) I_{k}\left(x\left(t_{k}^{-}\right)\right), \quad t \in\left(t_{m}, b\right],
\end{array}\right.
$$

where $\mathscr{T}(\cdot)$ and $\delta(\cdot)$ are called characteristic solution operators and are given by

$$
\begin{aligned}
& \mathscr{T}(t)=\int_{0}^{\infty} \xi_{q}(\theta) T\left(t^{q} \theta\right) d \theta, \\
& \mathcal{S}(t)=q \int_{0}^{\infty} \theta \xi_{q}(\theta) T\left(t^{q} \theta\right) d \theta,
\end{aligned}
$$

and, for $\theta \in(0, \infty)$,

$$
\xi_{q}(\theta)=\frac{1}{q} \theta^{-1-1 / q} \varpi_{q}\left(\theta^{-1 / q}\right) \geq 0,
$$

where $\xi_{q}$ is a probability density function defined on $(0, \infty)$; that is,

$$
\begin{aligned}
\xi_{q}(\theta) & \geq 0, \quad \theta \in(0, \infty), \\
\int_{0}^{\infty} \xi_{q}(\theta) d \theta & =1 .
\end{aligned}
$$

Definition 7 (see [21]). By a PC-mild solution of system (4), we mean a function $x \in \mathrm{PC}[I, \mathbb{X}]$ that satisfies the following integral equation:

$$
\begin{aligned}
& x(t) \\
& =\left\{\begin{array}{l}
\mathscr{T}(t)\left[x_{0}-g(x)\right]+\int_{0}^{t}(t-s)^{q-1} \mathcal{S}(t-s)[f(s, x(s),(H x)(s))+B u(s)] d s, \quad t \in\left[0, t_{1}\right], \\
\mathscr{T}(t)\left[x_{0}-g(x)\right]+\int_{0}^{t}(t-s)^{q-1} \mathcal{S}(t-s)[f(s, x(s),(H x)(s))+B u(s)] d s+\mathscr{T}\left(t-t_{1}\right) I_{1}\left(x\left(t_{1}^{-}\right)\right), \quad t \in\left(t_{1}, t_{2}\right], \\
\vdots \\
\mathscr{T}(t)\left[x_{0}-g(x)\right]+\int_{0}^{t}(t-s)^{q-1} \mathcal{S}(t-s)[f(s, x(s),(H x)(s))+B u(s)] d s+\sum_{k=1}^{m} \mathscr{T}\left(t-t_{k}\right) I_{k}\left(x\left(t_{k}^{-}\right)\right), \quad t \in\left(t_{m}, b\right] .
\end{array}\right.
\end{aligned}
$$

Definition 8. System (4) is said to be controllable on the interval $I$ if, for every $x_{0}, x_{1} \in \mathbb{X}$, there exists a control $u \in L^{2}[I, U]$ such that a mild solution $x$ of (4) satisfies $x(b)+g(x)=x_{1}$.

Lemma 9 (see [20]). Linear operator $A$ is the infinitesimal generator of a uniformly continuous semigroup if and only if $A$ is a bounded linear operator.

Lemma 10 (see [13] Krasnoselskii's fixed point theorem). Let $\mathbb{X}$ be a Banach space, let $B$ be a bounded closed and convex subset of $\mathbb{X}$, and let $F_{1}, F_{2}$ be maps of $B$ into $\mathbb{X}$ such that $F_{1} x+$ $F_{2} y \in B$ for every pair $x, y \in B$. If $F_{1}$ is a contraction and $F_{2}$ is completely continuous, then the equation $F_{1} x+F_{2} x=x$ has a solution in $B$

Lemma 11 (see $[22,23])$. The operators $\mathscr{T}(t)$ and $\mathcal{S}(t)$ defined by (9) have the following properties: (i) For any fixed $t \geq 0, \mathscr{T}(t)$ and $\delta(t)$ are linear and bounded operators; that is, for any $x \in \mathbb{X}$,

$$
\begin{aligned}
\|\mathscr{T}(t) x\| & \leq M_{1}\|x\|, \\
\|\mathcal{S}(t) x\| & \leq \frac{q M_{1}}{\Gamma(1+q)}\|x\| .
\end{aligned}
$$

(ii) $\{\mathscr{T}(t), t \geq 0\}$ and $\{\mathcal{S}(t), t \geq 0\}$ are strongly continuous.

(iii) $\{\mathscr{T}(t), t \geq 0\}$ and $\{\delta(t), t \geq 0\}$ are uniformly continuous.

Remark 12. Since the infinitesimal generator $A$ is a linear bounded operator and thanks to Definition 5 and Lemma 9, we can get that (iii) is satisfied.

Lemma 13 (see [21]). For $\sigma \in(0,1]$ and $0<a \leq b,\left|a^{\sigma}-b^{\sigma}\right| \leq$ $(b-a)^{\sigma}$. 


\section{Existence and Uniqueness of PC-Mild Solutions}

In order to prove the existence and uniqueness of mild solutions of (3), we have the following assumptions:

$\left(\mathbf{H}_{1}\right) f: I \times \mathbb{X} \times \mathbb{X} \rightarrow \mathbb{X}$ is continuous and there exist two functions $\mu_{1}, \mu_{2} \in L\left[I, \mathbb{R}^{+}\right]$such that

$$
\begin{aligned}
& \left\|f\left(t, x_{1}, y_{1}\right)-f\left(t, x_{2}, y_{2}\right)\right\| \\
& \quad \leq \mu_{1}(t)\left\|x_{1}-x_{2}\right\|+\mu_{2}(t)\left\|y_{1}-y_{2}\right\|,
\end{aligned}
$$

$$
x_{1}, x_{2}, y_{1}, y_{2} \in \mathbb{X}
$$

$\left(\mathbf{H}_{2}\right) h: \Delta \times \mathbb{X} \rightarrow \mathbb{X}$ is continuous and there exists a function $v_{1} \in C\left[I, \mathbb{R}^{+}\right]$such that

$$
\begin{array}{r}
\left\|h\left(t, s, x_{1}\right)-h\left(t, s, x_{2}\right)\right\| \leq v_{1}(t)\left\|x_{1}-x_{2}\right\|, \\
x_{1}, x_{2} \in \mathbb{X} .
\end{array}
$$

$\left(\mathbf{H}_{3}\right) I_{k}: \mathbb{X} \rightarrow \mathbb{X}$ are continuous and there exist $\omega_{k} \epsilon$ $C\left[I, \mathbb{R}^{+}\right]$such that

$$
\begin{array}{r}
\left\|I_{k}\left(x_{1}\right)-I_{k}\left(x_{2}\right)\right\| \leq \omega_{k}(t)\left\|x_{1}-x_{2}\right\|, \\
x_{1}, x_{2} \in \mathbb{X}, k=1,2, \ldots, m .
\end{array}
$$

$\left(\mathbf{H}_{4}\right) \mathrm{g}$ is continuous and there exists a function $\phi \in$ $C\left[I, \mathbb{R}^{+}\right]$such that

$$
\left\|g\left(x_{1}\right)-g\left(x_{2}\right)\right\| \leq \phi(t)\left\|x_{1}-x_{2}\right\| .
$$

$\left(\mathbf{H}_{5}\right)$ The function $\Omega_{m}(t): I \rightarrow \mathbb{R}^{+}$is defined by

$$
\begin{aligned}
\Omega_{m}(t)= & m \omega_{0} M_{1}+M_{1} \phi(t)+\frac{q M_{1}}{\Gamma(1+q)} \\
& \times \int_{0}^{t}(t-s)^{q-1}\left(\mu_{1}(s)+\nu_{1}^{0} b \mu_{2}(s)\right) d s,
\end{aligned}
$$

where $\nu_{1}^{0}=\max \left\{\nu_{1}(t) \mid t \in I\right\}, \omega_{0}=\max \left\{\omega_{k}(t) \mid t \epsilon\right.$ $I, k=1,2, \ldots, m\}$, and $0<\Omega_{m}(t)<1, t \in I$.

$\left(\mathbf{H}_{5}^{\prime}\right)$ The constant $\Omega_{u}$ and function $\Omega_{m}^{\prime}(t): I \rightarrow \mathbb{R}^{+}$are defined by

$$
\begin{aligned}
\Omega_{u}= & \omega_{0} m M_{1}+\phi_{0} M_{1}+\frac{q K M_{1}}{\Gamma(1+q)} \\
& \times \int_{0}^{b}(b-s)^{q-1}\left(\mu_{1}(s)+\nu_{1}^{0} b \mu_{2}(s)\right) d s, \\
\Omega_{m}^{\prime}(t)= & \omega_{0} m M_{1}+\phi_{0} M_{1}+\frac{q M_{1}}{\Gamma(1+q)} \\
& \times \int_{0}^{t}(t-s)^{q-1}\left(\mu_{1}(t)+\nu_{1}^{0} b \mu_{2}(t)\right) d s \\
& +\frac{q M_{1} \Omega_{u}}{\Gamma(1+q)} \int_{0}^{t}(t-s)^{q-1} d s,
\end{aligned}
$$

where $\phi_{0}=\max \{\phi(t) \mid t \in I\}$ and $0<\Omega_{m}^{\prime}(t)<1$, $t \in I$.

Theorem 14. If hypotheses $\left(\mathbf{H}_{1}\right)-\left(\mathbf{H}_{5}\right)$ are satisfied, then (3) has a unique PC-mild solution.

Proof. Define the operator $Q$ on $\mathrm{PC}[I, \mathbb{X}]$ by

$(\mathrm{Q} x)(t)$

$$
=\left\{\begin{array}{l}
\mathscr{T}(t)\left[x_{0}-g(x)\right]+\int_{0}^{t}(t-s)^{q-1} \mathcal{S}(t-s) f(s, x(s),(H x)(s)) d s, \quad t \in\left[0, t_{1}\right], \\
\mathscr{T}(t)\left[x_{0}-g(x)\right]+\int_{0}^{t}(t-s)^{q-1} \mathcal{S}(t-s) f(s, x(s),(H x)(s)) d s+\mathscr{T}\left(t-t_{1}\right) I_{1}\left(x\left(t_{1}^{-}\right)\right), \quad t \in\left(t_{1}, t_{2}\right], \\
\vdots \\
\mathscr{T}(t)\left[x_{0}-g(x)\right]+\int_{0}^{t}(t-s)^{q-1} \mathcal{S}(t-s) f(s, x(s),(H x)(s)) d s+\sum_{k=1}^{m} \mathscr{T}\left(t-t_{k}\right) I_{k}\left(x\left(t_{k}^{-}\right)\right), \quad t \in\left(t_{m}, b\right] .
\end{array}\right.
$$

For $0 \leq \tau<t \leq t_{1}$, by virtue of (20), we conclude that

$$
\begin{aligned}
& \|(Q x)(t)-(Q x)(\tau)\| \leq\|\mathscr{T}(t)-\mathscr{T}(\tau)\|\left\|x_{0}-g(x)\right\| \\
& +\left\|\int_{\tau}^{t}(t-s)^{q-1} \mathcal{S}(t-s) f(s, x(s),(H x)(s)) d s\right\| \\
& +\| \int_{0}^{\tau}(t-s)^{q-1}[\mathcal{S}(t-s)-\mathcal{S}(\tau-s)] \\
& \quad \times f(s, x(s),(H x)(s)) d s \|
\end{aligned}
$$

$$
\begin{aligned}
& +\| \int_{0}^{\tau}\left[(t-s)^{q-1}-(\tau-s)^{q-1}\right] \mathcal{S}(\tau-s) \\
& \times f(s, x(s),(H x)(s)) d s \| .
\end{aligned}
$$

It follows from Lemma 11, part (iii) and Lemma 13 that

$$
\|(Q x)(t)-(Q x)(\tau)\| \longrightarrow 0 \quad \text { as } t \longrightarrow \tau .
$$


Thus, we deduce that $Q x \in C\left[\left[0, t_{1}\right], \mathbb{X}\right]$. For $t_{1}<\tau<t \leq t_{2}$, we have

$$
\begin{aligned}
& \|(Q x)(t)-(Q x)(\tau)\| \leq\|\mathscr{T}(t)-\mathscr{T}(\tau)\|\left\|x_{0}-g(x)\right\| \\
& +\left\|\mathscr{T}\left(t-t_{1}\right)-\mathscr{T}\left(\tau-t_{1}\right)\right\|\left\|I_{1}\left(x\left(t_{1}^{-}\right)\right)\right\| \\
& +\| \int_{\tau}^{t}(t-s)^{q-1} \mathcal{S}(t-s) \\
& \times f(s, x(s),(H x)(s)) d s \| \\
& +\| \int_{0}^{\tau}(t-s)^{q-1}[\mathcal{S}(t-s)-\mathcal{S}(\tau-s)] \\
& \times f(s, x(s),(H x)(s)) d s \| \\
& +\| \int_{0}^{\tau}\left[(t-s)^{q-1}-(\tau-s)^{q-1}\right] \mathcal{S}(\tau-s) \\
& \times f(s, x(s),(H x)(s)) d s \| .
\end{aligned}
$$

From (23), we know that $Q x \in C\left[\left(t_{1}, t_{2}\right], \mathbb{X}\right]$. Using the same method, we obtain $Q x \in C\left[\left(t_{2}, t_{3}\right], \mathbb{X}\right], \ldots, Q x \in$
$C\left[\left(t_{m}, b\right], \mathbb{X}\right]$, and therefore $Q x \in \operatorname{PC}[I, \mathbb{X}]$. For each $t \in$ $\left(t_{i}, t_{i+1}\right], 1 \leq i \leq m, x, y \in \mathrm{PC}[I, \mathbb{X}]$,

$$
\begin{aligned}
& \|(Q x)(t)-(Q y)(t)\| \leq M_{1} \phi(t)+\frac{q M_{1}}{\Gamma(1+q)} \int_{0}^{t}(t \\
& -s)^{q-1} \times\left(\mu_{1}(s)+v_{1}^{0} b \mu_{2}(s)\right) d s\|x-y\|_{\mathrm{PC}} \\
& +\| \sum_{k=1}^{i} \mathscr{T}\left(t-t_{i}\right) I_{k}\left(x\left(t_{k}^{-}\right)\right) \\
& -\sum_{k=1}^{i} \mathscr{T}\left(t-t_{i}\right) I_{k}\left(y\left(t_{k}^{-}\right)\right)\left\|\leq \Omega_{i}(t)\right\| x-y \|_{\mathrm{PC}} .
\end{aligned}
$$

When $i=m$, we get

$$
\|(Q x)(t)-(Q y)(t)\| \leq \Omega_{m}(t)\|x-y\|_{\mathrm{PC}} .
$$

It follows now from $\Omega_{i}(t) \leq \Omega_{m}(t),\left(\mathbf{H}_{5}\right)$, and the contraction mapping principle that $Q$ has a unique fixed point $x \in$ $\mathrm{PC}[I, \mathbb{X}]$; that is,

$$
x(t)=\left\{\begin{array}{l}
\mathscr{T}(t)\left[x_{0}-g(x)\right]+\int_{0}^{t}(t-s)^{q-1} \mathcal{S}(t-s) f(s, x(s),(H x)(s)) d s, \quad t \in\left[0, t_{1}\right], \\
\mathscr{T}(t)\left[x_{0}-g(x)\right]+\int_{0}^{t}(t-s)^{q-1} \mathcal{S}(t-s) f(s, x(s),(H x)(s)) d s+\mathscr{T}\left(t-t_{1}\right) I_{1}\left(x\left(t_{1}^{-}\right)\right), \quad t \in\left(t_{1}, t_{2}\right], \\
\vdots \\
\mathscr{T}(t)\left[x_{0}-g(x)\right]+\int_{0}^{t}(t-s)^{q-1} \mathcal{S}(t-s) f(s, x(s),(H x)(s)) d s+\sum_{k=1}^{m} \mathscr{T}\left(t-t_{k}\right) I_{k}\left(x\left(t_{k}^{-}\right)\right), \quad t \in\left(t_{m}, b\right],
\end{array}\right.
$$

is a unique PC-mild solution of (3). The proof is complete.

In order to obtain more existence results, we have the following assumptions:

$\left(\mathbf{H}_{6}\right) f: I \times \mathbb{X} \times \mathbb{X} \rightarrow \mathbb{X}$ is continuous and there exist three functions $\mu_{3}, \mu_{4}, \mu_{5} \in L\left[I, \mathbb{R}^{+}\right]$such that

$$
\|f(t, x, y)\| \leq \mu_{3}(t)+\mu_{4}(t)\|x\|+\mu_{5}(t)\|y\|,
$$

$$
t \in I, x, y \in \mathbb{X} \text {. }
$$

$\left(\mathbf{H}_{7}\right) h: \Delta \times \mathbb{X} \rightarrow \mathbb{X}$ is continuous and there exist two functions $\nu_{2}, \nu_{3} \in C\left[I, \mathbb{R}^{+}\right]$such that

$$
\|h(t, s, x)\| \leq \nu_{2}(s)+v_{3}(s)\|x\|, \quad x \in \mathbb{X} .
$$

$\left(\mathbf{H}_{8}\right) I_{k}: \mathbb{X} \rightarrow \mathbb{X}$ are continuous and there exist $\psi_{k} \in$ $C\left[I, \mathbb{R}^{+}\right]$such that

$$
\left\|I_{k}(x)\right\| \leq \psi_{k}(t)\|x\|, \quad x \in \mathbb{X} .
$$

Define $\psi_{0}=\max \left\{\psi_{k}(t) \mid t \in I, k=1,2, \ldots, m\right\}$.
$\left(\mathbf{H}_{9}\right)$ There exists a function $\kappa \in C\left[I, \mathbb{R}^{+}\right]$such that

$$
\|g(x)\| \leq \kappa(t)\|x\|, \quad x \in \mathbb{X} .
$$

Define $\kappa_{0}=\max \{\kappa(t) \mid t \in I\}$.

$\left(\mathbf{H}_{10}\right)$ For all bounded subsets $B_{r}$, the set

$$
\begin{gathered}
\Pi_{m, h, \delta}(t)=\left\{\int_{0}^{t-h}(t-s)^{q-1} \delta_{\delta}(t-s) F(s) d s\right. \\
\left.+\sum_{k=1}^{m} \mathscr{T}_{\delta}\left(t-t_{k}\right) I_{k}\left(x\left(t_{k}^{-}\right)\right): x \in B_{r}\right\}
\end{gathered}
$$

is relatively compact in $\mathbb{X}$ for arbitrary $h \in(0, t)$ and $\delta>0$, where $\mathscr{T}_{\delta}(t)$ and $\mathcal{S}_{\delta}(t)$ are defined by

$$
\begin{aligned}
& \mathscr{T}_{\delta}(t)=\int_{\delta}^{\infty} \xi_{q}(\theta) T\left(t^{q} \theta\right) d \theta, \\
& \mathcal{S}_{\delta}(t)=q \int_{\delta}^{\infty} \theta \xi_{q}(\theta) T\left(t^{q} \theta\right) d \theta .
\end{aligned}
$$


$\left(\mathbf{H}_{10}^{\prime}\right)$ For all bounded subsets $B_{r}$, the set

$$
\begin{aligned}
\Pi_{m, h, \delta}^{\prime}(t) \\
\quad=\left\{\int_{0}^{t-h}(t-s)^{q-1} \delta_{\delta}(t-s)[F(s)+B u(s)] d s\right. \\
\left.+\sum_{k=1}^{m} \mathscr{T}_{\delta}\left(t-t_{k}\right) I_{k}\left(x\left(t_{k}^{-}\right)\right): x \in B_{r}\right\}
\end{aligned}
$$

is relatively compact in $\mathbb{X}$ for arbitrary $h \in(0, t)$ and $\delta>0$.

Theorem 15. Let hypotheses $\left(\mathbf{H}_{4}\right)$ and $\left(\mathbf{H}_{6}\right)-\left(\mathbf{H}_{10}\right)$ be satisfied. If the inequalities

$$
\begin{array}{r}
\frac{q b^{q} M_{1}}{\Gamma(1+q)} \int_{0}^{b} \varphi_{2}(s) d s+m M_{1} \psi_{0}+M_{1} \kappa_{0}<1, \\
\phi_{0} M_{1}<1
\end{array}
$$

hold, where $\varphi_{2}(s)=\mu_{4}(s)+\mu_{5}(s) \int_{0}^{s} \nu_{3}(\theta) d \theta$ and $\phi_{0}$ is as in $\left(\mathbf{H}_{5}^{\prime}\right)$, then (3) has at least one PC-mild solution.

Proof. We shall present the results in six steps.

Step 1 (Continuity of $Q$ defined by (20) on $\left(t_{i}, t_{i+1}\right](i=$ $0,1,2, \ldots, m))$. Let $x_{n}, x \in \mathrm{PC}[I, \mathbb{X}]$ and $\left\|x_{n}-x^{*}\right\|_{\mathrm{PC}} \rightarrow$ $0(n \rightarrow \infty)$. Then $r=\sup _{n}\left\|x_{n}\right\|_{\mathrm{PC}}<\infty$ and $\left\|x^{*}\right\|_{\mathrm{PC}}<r$. For $t \in\left(t_{i}, t_{i+1}\right](i=0,1,2, \ldots, m)$, we have

$$
\begin{aligned}
& \left\|Q x_{n}(t)-Q x(t)\right\| \leq \frac{q M_{1}}{\Gamma(1+q)} \int_{0}^{t}(t-s)^{q-1} \\
& \quad \times \| f\left(s, x_{n}(s),\left(H x_{n}\right)(s)\right) \\
& \quad-f(s, x(s),(H x)(s)) \| d s \\
& +\psi_{0} M_{1} \sum_{k=1}^{m}\left\|I_{k}\left(x_{n}\left(t_{k}^{-}\right)\right)-I_{k}\left(x\left(t_{k}^{-}\right)\right)\right\| \\
& +M_{1}\left\|g\left(x_{n}\right)-g(x)\right\| .
\end{aligned}
$$

Since the functions $f, I_{k}$, and $g$ are continuous, we conclude that

$$
\begin{aligned}
f\left(s, x_{n}(s),\left(H x_{n}\right)(s)\right) & \longrightarrow f(s, x(s),(H x)(s)), \\
g\left(x_{n}\right) & \longrightarrow g(x), \\
I_{k}\left(x_{n}\left(t_{k}^{-}\right)\right) & \longrightarrow I_{k}\left(x\left(t_{k}^{-}\right)\right), \quad n \longrightarrow \infty .
\end{aligned}
$$

Applications of $\left(\mathbf{H}_{6}\right)$ and $\left(\mathbf{H}_{7}\right)$ yield

$$
\begin{gathered}
\left\|f\left(s, x_{n}(s),\left(H x_{n}\right)(s)\right)-f(s, x(s),(H x)(s))\right\| \\
\leq 2 \mu_{3}(s)+2 \mu_{5}(s) \int_{0}^{s} v_{2}(\theta) d \theta \\
\quad+\left(2 \mu_{4}(s)+2 \mu_{5}(s) \int_{0}^{s} v_{3}(\theta) d \theta\right) r,
\end{gathered}
$$

which implies that

$$
\begin{aligned}
& (t-s)^{q-1} \\
& \cdot\left\|f\left(s, x_{n}(s),\left(H x_{n}\right)(s)\right)-f(s, x(s),(H x)(s))\right\| \\
& \quad \in L^{1}\left[I, \mathbb{R}^{+}\right] .
\end{aligned}
$$

By Lebesgue's dominated convergence theorem, we get

$$
\begin{gathered}
\int_{0}^{t}(t-s)^{q-1} \times \| f\left(s, x_{n}(s), H x_{n}(s)\right) \\
-f(s, x(s), H x(s)) \| d s \longrightarrow 0,
\end{gathered}
$$

and so

$$
\lim _{n \rightarrow \infty}\left\|Q x_{n}(t)-Q x(t)\right\|_{\mathrm{PC}}=0 .
$$

Step 2 (Q maps bounded sets into bounded sets in $\mathrm{PC}[I, \mathbb{X}]$ ). From (20), we get

$$
\begin{aligned}
& \|(Q x)(t)\| \\
& =\left\|\mathscr{T}(t)\left[x_{0}-g(x)\right]\right\| \\
& \quad+\int_{0}^{t}(t-s)^{q-1}\|\mathcal{S}(t-s) f(s, x(s),(H x)(s))\| d s \\
& \quad+\sum_{k=1}^{m}\left\|\mathscr{T}\left(t-t_{k}\right) I_{k}\left(x\left(t_{k}^{-}\right)\right)\right\|,
\end{aligned}
$$

where

$$
\begin{aligned}
& \|f(s, x(s),(H x)(s))\| \\
& \leq \mu_{3}(s)+\mu_{5}(s) \int_{0}^{s} v_{2}(\theta) d \theta \\
& \quad+\left(\mu_{4}(s)+\mu_{5}(s) \int_{0}^{s} \nu_{3}(\theta) d \theta\right)\|x\| \\
& \leq \varphi_{1}(s)+\varphi_{2}(s)\|x\| .
\end{aligned}
$$

By Lemma 11 and (42), we obtain

$$
\begin{aligned}
\|(Q x)(t)\| \leq & \frac{q b^{q} M_{1}}{\Gamma(1+q)} \int_{0}^{t}\left(\varphi_{1}(s)+\varphi_{2}(s)\|x\|\right) d s \\
& +M_{1}\left\|x_{0}\right\|+M_{1} \kappa_{0}\|x\|+m M_{1} \psi_{0}\|x\| .
\end{aligned}
$$

Thus, for any $x \in B_{r}=\left\{x \in \mathrm{PC}[I, \mathbb{X}]:\|x\|_{\mathrm{PC}} \leq r\right\}$, we have

$\|(Q x)(t)\|$

$$
\begin{aligned}
\leq & M_{1}\left\|x_{0}\right\|+\frac{q b^{q} M_{1}}{\Gamma(1+q)} \int_{0}^{b} \varphi_{1}(s) d s \\
& +\left(\frac{q b^{q} M_{1}}{\Gamma(1+q)} \int_{0}^{b} \varphi_{2}(s) d s+m M_{1} \psi_{0}+M_{1} \kappa_{0}\right) r \\
= & \gamma_{1} .
\end{aligned}
$$


Hence, we deduce that $\|(Q x)(t)\| \leq \gamma_{1}$, that is, $Q$ maps bounded sets into bounded sets in PC $[I, \mathbb{X}]$.

Step $3\left(Q\left(B_{r}\right)\right.$ is equicontinuous with $B_{r}$ on $\left(t_{i}, t_{i+1}\right](i=$ $0,1,2, \ldots, m))$. For any $x \in B_{r}, t^{\prime}, t^{\prime \prime} \in\left(t_{i}, t_{i+1}\right](i=$ $0,1,2, \ldots, m)$, we obtain

$$
\begin{aligned}
& \left\|(Q x)\left(t^{\prime \prime}\right)-(Q x)\left(t^{\prime}\right)\right\| \leq\left\|\mathscr{T}\left(t^{\prime \prime}\right) x_{0}-\mathscr{T}\left(t^{\prime}\right) x_{0}\right\| \\
& +\left\|\mathscr{T}\left(t^{\prime \prime}\right) g(x)-\mathscr{T}\left(t^{\prime}\right) g(x)\right\| \\
& +\| \int_{0}^{t^{\prime \prime}}\left(t^{\prime \prime}-s\right)^{q-1} \mathcal{S}\left(t^{\prime \prime}-s\right) F(s) d s \\
& \quad-\int_{0}^{t^{\prime}}\left(t^{\prime}-s\right)^{q-1} \mathcal{S}\left(t^{\prime}-s\right) F(s) d s \| \\
& +\| \sum_{k=1}^{m} \mathscr{T}\left(t^{\prime \prime}-t_{k}\right) I_{k}\left(x\left(t_{k}^{-}\right)\right) \\
& \quad-\sum_{k=1}^{m} \mathscr{T}\left(t^{\prime}-t_{k}\right) I_{k}\left(x\left(t_{k}^{-}\right)\right) \| .
\end{aligned}
$$

Based on a straightforward computation, we have

$$
\begin{aligned}
& \left\|(Q x)\left(t^{\prime \prime}\right)-(Q x)\left(t^{\prime}\right)\right\| \leq\left\|\mathscr{T}\left(t^{\prime \prime}\right)-\mathscr{T}\left(t^{\prime}\right)\right\|\left\|x_{0}\right\| \\
& +\left\|\mathscr{T}\left(t^{\prime \prime}\right) g(x)-\mathscr{T}\left(t^{\prime}\right) g(x)\right\| \\
& +\left\|\int_{t^{\prime}}^{t^{\prime \prime}}\left(t^{\prime \prime}-s\right)^{q-1} \mathcal{S}\left(t^{\prime \prime}-s\right) F(s) d s\right\| \\
& +\| \int_{0}^{t^{\prime}}\left[\left(t^{\prime \prime}-s\right)^{q-1}-\left(t^{\prime}-s\right)^{q-1}\right] \mathcal{S}\left(t^{\prime \prime}-s\right) \\
& \cdot F(s) d s\|+\| \int_{0}^{t^{\prime}}\left(t^{\prime}-s\right)^{q-1} \\
& \cdot\left[\mathcal{S}\left(t^{\prime \prime}-s\right)-\mathcal{S}\left(t^{\prime}-s\right)\right] F(s) d s \| \\
& +m M_{1}\left\|\mathscr{T}\left(t^{\prime \prime}-t^{\prime}\right)-I\right\|\left\|I_{k}\left(x\left(t_{k}^{-}\right)\right)\right\| .
\end{aligned}
$$

It follows from Lemma 11, part (iii) and Lemma 13 that $\lim _{t^{\prime \prime} \rightarrow t^{\prime}}\left\|(Q x)\left(t^{\prime \prime}\right)-(Q x)\left(t^{\prime}\right)\right\|=0$. Thus, $Q\left(B_{r}\right)$ is equicontinuous with $B_{r}$ on $\left(t_{i}, t_{i+1}\right](i=0,1,2, \ldots, m)$.

Step $4\left(P_{i}\right.$ map $B_{r}$ into a precompact set in $\left.\mathbb{X}(i=1, \ldots, m)\right)$. We define the operator

$$
(Q x)(t)=\left(P_{i} x\right)(t)+(L x)(t),
$$

where

$$
\begin{aligned}
& (L x)(t)=\mathscr{T}(t)\left[x_{0}-g(x)\right], \\
& \left(P_{i} x\right)(t) \\
& =\int_{0}^{t}(t-s)^{q-1} \mathcal{S}(t-s) f(s, x(s),(H x)(s)) d s \\
& \quad+\sum_{k=1}^{i} \mathscr{T}\left(t-t_{k}\right) I_{k}\left(x\left(t_{k}^{-}\right)\right), \quad i=1, \ldots, m .
\end{aligned}
$$
Set

Define $\Pi=P_{i} B_{r}$ and $\Pi(t)=\left\{\left(P_{i} x\right)(t): x \in B_{r}\right\}$ for $t \in I$.

$$
\Pi_{i, h, \delta}(t)=\left\{\left(P_{i, h, \delta} x\right)(t): x \in B_{r}\right\},
$$

where

$$
\begin{gathered}
\Pi_{i, h, \delta}(t)=\left\{\int_{0}^{t-h}(t-s)^{q-1} \mathcal{S}_{\delta}(t-s) F(s) d s\right. \\
\left.+\sum_{k=1}^{i} \mathscr{T}_{\delta}\left(t-t_{k}\right) I_{k}\left(x\left(t_{k}^{-}\right)\right): x \in B_{r}\right\} .
\end{gathered}
$$

From hypotheses we imposed and the same method used in [16, Theorem 3.2], it is not difficult to verify that the set $\Pi(t)$ can be arbitrary approximated by the relatively compact set $\Pi_{i, h, \delta}(t)$. Thus, $P_{i}\left(B_{r}\right)(t)$ are relatively compact in $\mathbb{X}$.

Step $5\left(L x+P_{i} y \in B_{r}\right.$ for $\left.x, y \in B_{r}(i=1, \ldots, m)\right)$. Note that

$$
\frac{q b^{q} M_{1}}{\Gamma(1+q)} \int_{0}^{b} \varphi_{2}(s) d s+m M_{1} \psi_{0}+M_{1} \kappa_{0}<1 .
$$

Choose

$$
\begin{aligned}
& \frac{M_{1}\left\|x_{0}\right\|+\left(q b^{q} M_{1} / \Gamma(1+q)\right) \int_{0}^{b} \varphi_{1}(s) d s}{1-\left(q b^{q} M_{1} / \Gamma(1+q)\right) \int_{0}^{b} \varphi_{2}(s) d s-m M_{1} \psi_{0}-M_{1} \kappa_{0}} \\
& \leq r
\end{aligned}
$$

and define operators $L$ and $P_{i}$ on $B_{r}$ by

$$
\begin{aligned}
& (L x)(t)=\mathscr{T}(t)\left[x_{0}-g(x)\right], \\
& \left(P_{i} x\right)(t) \\
& =\int_{0}^{t}(t-s)^{q-1} \mathcal{S}(t-s) f(s, x(s),(H x)(s)) d s \\
& \quad+\sum_{k=1}^{i} \mathscr{T}\left(t-t_{k}\right) I_{k}\left(x\left(t_{k}^{-}\right)\right), \quad i=1, \ldots, m .
\end{aligned}
$$

It is sufficient to proceed exactly as in step 1 to step 4 of the proof to deduce that $P_{i}$ are continuous and compact. Thus, to complete this proof, it suffices to show that $L$ is a contraction 
mapping and that $L x+P_{i} y \in B_{r}$ for $x, y \in B_{r}$. Indeed, for any $x \in B_{r}$, by virtue of (43) and (51), we have

$$
\begin{aligned}
& \|(Q x)(t)\| \\
& \quad \leq M_{1}\left\|x_{0}\right\|+\frac{q b^{q} M_{1}}{\Gamma(1+q)} \int_{0}^{b} \varphi_{1}(s) d s \\
& \quad+\left(\frac{q b^{q} M_{1}}{\Gamma(1+q)} \int_{0}^{b} \varphi_{2}(s) d s+m M_{1} \psi_{0}+M_{1} \kappa_{0}\right) r
\end{aligned}
$$

$\leq r$.

Consequently, if $x, y \in B_{r}$, then $L x+P_{i} y \in B_{r}$.

Step 6 ( $L$ is a contraction mapping). For any $t^{\prime}, t^{\prime \prime} \epsilon$ $\left(t_{i}, t_{i+1}\right](i=0,1,2, \ldots, m)$ and $x, y \in \operatorname{PC}[I, \mathbb{X}]$, we have

$$
\begin{aligned}
\|(L x)(t)-(L y)(t)\| & \leq\|\mathscr{T}(t)(g(x)-g(y))\| \\
& \leq\|\mathscr{T}(t)\|\|g(x)-g(y)\| \\
& \leq \phi_{0} M_{1}\|x-y\|_{\mathrm{PC}} .
\end{aligned}
$$

Since $\phi_{0} M_{1}<1, L$ is a contraction mapping. Hence, by Lemma 10, we conclude that (3) has at least one PC-mild solution on $I$. This completes the proof.

\section{Controllability Results}

In this section, we impose the following conditions to prove the results.

$\left(\mathbf{H}_{11}\right)$ Define $I_{i}=\left(t_{i-1}, t_{i}\right](i=1,2, \ldots, m+1)$. The linear operator $W_{i}$ from $L^{2}\left[I_{i}, U\right]$ into $\mathbb{X}$ defined by

$$
W_{i} u=\int_{0}^{t_{i}}\left(t_{i}-s\right)^{q-1} \mathcal{S}\left(t_{i}-s\right) B u(s) d s
$$

induces an invertible operator $\widetilde{W}_{i}^{-}$defined on $L^{2}\left[I_{i}, U\right] / \operatorname{Ker}_{i}$ and there exists a positive constant $K>0$ such that $\left\|B \widetilde{W}_{i}^{-}\right\| \leq K$.

Theorem 16. If hypotheses $\left(\mathbf{H}_{1}\right)-\left(\mathbf{H}_{4}\right),\left(\mathbf{H}_{5}^{\prime}\right)$, and $\left(\mathbf{H}_{11}\right)$ are satisfied, then system (4) is controllable on I.

Proof. Using $\left(\mathbf{H}_{11}\right)$, for an arbitrary function $x(\cdot)$, we define the piecewise continuous control $u$ by

$u(t)$

$$
=\left\{\begin{array}{l}
\widetilde{W}_{1}^{-}\left[x_{0}+\frac{x_{1}-x_{0}}{m+1}-\mathscr{T}\left(t_{1}\right)\left[x_{0}-g(x)\right]-\int_{0}^{t_{1}}\left(t_{1}-s\right)^{q-1} \mathcal{S}\left(t_{1}-s\right) f(s, x(s),(H x)(s)) d s\right](t), \quad t \in\left[0, t_{1}\right], \\
\widetilde{W}_{2}^{-}\left[x_{0}+\frac{2\left(x_{1}-x_{0}\right)}{m+1}-\mathscr{T}\left(t_{2}\right)\left[x_{0}-g(x)\right]-\int_{0}^{t_{2}}\left(t_{2}-s\right)^{q-1} \mathcal{S}\left(t_{2}-s\right) f(s, x(s),(H x)(s)) d s-\mathscr{T}\left(t_{2}-t_{1}\right) I_{1}\left(x\left(t_{1}^{-}\right)\right)\right](t), \quad t \in\left(t_{1}, t_{2}\right], \\
\vdots \\
\widetilde{W}_{m+1}^{-}\left[x_{1}-\mathscr{T}(b)\left[x_{0}-g(x)\right]-\int_{0}^{b}(b-s)^{q-1} \mathcal{S}(b-s) f(s, x(s),(H x)(s)) d s-\sum_{k=1}^{m} \mathscr{T}\left(b-t_{k}\right) I_{k}\left(x\left(t_{k}^{-}\right)\right)\right](t), \quad t \in\left(t_{m}, b\right] .
\end{array}\right.
$$

On the basis of this control, with a similar proof to

Theorem 14, we can conclude that the operator $Q$ defined by

$(Q x)(t)$

$$
=\left\{\begin{array}{l}
\mathscr{T}(t)\left[x_{0}-g(x)\right]+\int_{0}^{t}(t-s)^{q-1} \mathcal{S}(t-s)[f(s, x(s),(H x)(s))+B u(s)] d s, \quad t \in\left[0, t_{1}\right], \\
\mathscr{T}(t)\left[x_{0}-g(x)\right]+\int_{0}^{t}(t-s)^{q-1} \mathcal{S}(t-s)[f(s, x(s),(H x)(s))+B u(s)] d s+\mathscr{T}\left(t-t_{1}\right) I_{1}\left(x\left(t_{1}^{-}\right)\right), \quad t \in\left(t_{1}, t_{2}\right], \\
\vdots \\
\mathscr{T}(t)\left[x_{0}-g(x)\right]+\int_{0}^{t}(t-s)^{q-1} \mathcal{S}(t-s)[f(s, x(s),(H x)(s))+B u(s)] d s+\sum_{k=1}^{m} \mathscr{T}\left(t-t_{k}\right) I_{k}\left(x\left(t_{k}^{-}\right)\right), \quad t \in\left(t_{m}, b\right]
\end{array}\right.
$$


has a fixed point $x(\cdot)$. This fixed point is a PC-mild solution of system (4), which implies that the system is controllable on I. The proof is complete.

Theorem 17. Assume that hypotheses $\left(\mathbf{H}_{4}\right),\left(\mathbf{H}_{6}\right)-\left(\mathbf{H}_{9}\right),\left(\mathbf{H}_{10}^{\prime}\right)$, and $\left(\mathbf{H}_{11}\right)$ are satisfied. If the inequalities

$$
\begin{array}{r}
\frac{q b^{q} M_{1}}{\Gamma(1+q)} \int_{0}^{b}\left(\varphi_{2}(s)+N_{1}\right) d s+m M_{1} \psi_{0}+M_{1} \kappa_{0}<1 \\
\phi_{0} M_{1}<1
\end{array}
$$

hold, where $N_{1}=q K M_{1} \int_{0}^{b}(b-s)^{q-1} \varphi_{2}(s) d s / \Gamma(1+q)+$ $m K M_{1} \psi_{0}$ and $\varphi_{2}(s)$ and $\phi_{0}$ are as in Theorem 15 , then system (4) is controllable on $I$.

Proof. The proof is similar to that of Theorem 15 and so is omitted.

\section{Example}

Consider the following nonlinear partial integrodifferential equation of the form

$$
\begin{aligned}
& \frac{\partial^{1 / 3}}{\partial t^{1 / 3}} z(t, y) \\
& \quad=\int_{0}^{1}(y-s) z(s, y) d s+f(t, z(t, y), H z(t, y)) \\
& \quad+\mu(t, y), \\
& z(t, 0)=z(t, 1)=0, \quad t \in J=[0,1] \\
& z(0, y)+\phi(t) z(t, y)=z_{0}(y), \quad 0 \leq y \leq 1, \\
& \left.\Delta z\right|_{t=1 / 2}=I_{1}\left(x\left(\frac{1^{-}}{2}\right)\right),
\end{aligned}
$$

where $\mu: J \times(0,1) \rightarrow(0,1)$ is continuous. Let us take $\mathbb{X}=$ $C([0,1])$. Consider the operator $A: D(A) \subseteq \mathbb{X} \rightarrow \mathbb{X}$ defined by

$$
(A w)(t)=\int_{0}^{1}(y-s) w(s) d s
$$

It is not difficult to get

$$
\begin{aligned}
\|A w\| & =\|w\| \int_{0}^{1}|y-s| d s=\left(\frac{1}{2}-y(1-y)\right)\|w\| \\
& \leq \frac{1}{2}\|w\|
\end{aligned}
$$

and, clearly, $A$ is the infinitesimal generator of a uniformly continuous semigroup $(T(t))_{t \geq 0}$ on $\mathbb{X}$. Put $x(t)(y)=z(t, y)$ and $u(t)(y)=\mu(t, y)$, and take

$$
\begin{aligned}
f(t, x, H x) & =k_{0} x+H x, \\
(H x)(t) & =\int_{0}^{t} h(t, s, x(s)) d s, \\
h(t, s, x) & =k_{1} x, \\
I_{1}(x) & =\omega(t) x, \\
g(x) & =\phi(t) x,
\end{aligned}
$$

where $k_{0}$ and $k_{1}$ are positive constants and $\omega(t)$ and $\phi(t)$ are continuous functions. Then $f:[0,1] \times \mathbb{X} \times \mathbb{X} \rightarrow \mathbb{X}$ and $I_{1}: \mathbb{X} \rightarrow \mathbb{X}$ are continuous functions; $f, g, I_{1}$, and $h$ satisfy $\left(\mathbf{H}_{6}\right)-\left(\mathbf{H}_{9}\right)$, respectively.

For $y \in(0,1]$, we define

$$
\begin{aligned}
& W_{1} u=\int_{0}^{1 / 2}\left(\frac{1}{2}-s\right)^{-2 / 3} \mathcal{S}\left(\frac{1}{2}-s\right) B u(s) d s, \\
& W_{2} u=\int_{0}^{1}(1-s)^{-2 / 3} \mathcal{S}(1-s) B u(s) d s,
\end{aligned}
$$

where

$$
\begin{aligned}
& \mathscr{T}(t) w(s)=\int_{0}^{\infty} \xi_{1 / 3}(\theta) w\left(t^{1 / 3} \theta+s\right) d \theta, \\
& \mathcal{S}(t) w(s)=\frac{1}{3} \int_{0}^{\infty} \theta \xi_{1 / 3}(\theta) w\left(t^{1 / 3} \theta+s\right) d \theta,
\end{aligned}
$$

and, for $\theta \in(0, \infty)$,

$$
\begin{aligned}
& \xi_{1 / 3}(\theta)=3 \theta^{-4} \omega_{1 / 3}\left(\theta^{-3}\right), \\
& \omega_{1 / 3}(\theta) \\
& \quad=\frac{1}{\pi} \sum_{n=1}^{\infty}(-1)^{n-1} \theta^{-(n+3) / 3} \frac{\Gamma((n+3) / 3)}{n !} \sin \left(\frac{n \pi}{3}\right) .
\end{aligned}
$$

Moreover, the linear operator $W_{i}$ from $L^{2}\left[I_{i}, U\right](i=1,2)$ into $\mathbb{X}$ induces an invertible operator $\widetilde{W}_{i}^{-}$defined on $L^{2}\left[I_{i}, U\right] / \operatorname{Ker} W_{i}$ and there exists a positive constant $K>0$ such that $\left\|B \widetilde{W}_{i}^{-}\right\| \leq K$; that is, $\left(\mathbf{H}_{11}\right)$ is satisfied. With the choices of $A, f, g, H$, and $B=I$ (the identity operator), we see that (60) is an abstract formulation of (4). All conditions of Theorem 17 are able to be fulfilled, so we deduce that (60) is controllable on $I$. On the other hand, we have

$$
\begin{aligned}
& \|f(t, x, H x)-f(t, y, H y)\| \\
& \quad \leq k_{0}\|x-y\|+k_{1}\|x-y\|, \\
& \|h(t, s, x)-h(t, s, y)\| \leq k_{1}\|x-y\|, \\
& \left\|I_{1}(x)-I_{1}(y)\right\| \leq \omega(t)\|x-y\|, \\
& \|g(x)-g(y)\| \leq \phi(t)\|x-y\| .
\end{aligned}
$$

It is easy to see that all assumptions of Theorem 16 are satisfied when using the suitable choices of $k_{0}, k_{1}, \omega, \phi$. Hence, Theorem 16 can also yield controllability of (60) on $I$. 


\section{Conclusions}

In this paper, we studied the existence and uniqueness results for a class of impulsive fractional semilinear integrodifferential equations with nonlocal initial conditions in a Banach space. Introducing the concept of PC-mild solutions and using the piecewise continuous control functions and uniformly continuous semigroup, we obtained the controllability results for the corresponding fractional impulsive integrodifferential system. Assuming that the semigroup is compact and utilizing some additional conditions, Hernández and O'Regan [30] showed that some known results on exact controllability (see the references cited therein) are valid if and only if the Banach space is finite dimensional. Recently, Hernández et al. [31] pointed out that some recent results on exact controllability of abstract differential systems with an unbounded linear operator dominated by a sectorial operator were not applicable. Contrary to those results, we do not need in our results conflicting conditions, which, in a certain sense, is a significant improvement compared to the results in the cited papers. An illustrative example is given to demonstrate the effectiveness of the results obtained. Our future work will focus on constrained controllability, nonlocal problems, and their applications in nonlinear dynamical systems (see [3236]).

\section{Conflicts of Interest}

The authors declare that they have no conflicts of interest.

\section{Acknowledgments}

This research is supported by Shandong Provincial Natural Science Foundation (Grants nos. ZR2016AB04 and ZR2016JL021), a Project of Shandong Province Higher Educational Science and Technology Program (Grant no. J17KB121), Major International (Regional) Joint Research Project of National Natural Science Foundation of China (Grant no. 61320106011), National Natural Science Foundation of China (Grants nos. 61503171 and 61527809), China Postdoctoral Science Foundation (Grant no. 2015M582091), Foundation for Young Teachers of Qilu Normal University (Grants nos. 2016L0605, 2017JX2311, and 2017JX2312), Doctoral Scientific Research Foundation of Linyi University (Grant no. LYDX2015BS001), and Scientific Research Foundation for University Students of Qilu Normal University (Grant no. XS2017L05).

\section{References}

[1] A. Anguraj and K. Karthikeyan, "Existence of solutions for impulsive neutral functional differential equations with nonlocal conditions," Nonlinear Analysis: Theory, Methods \& Applications, vol. 70, no. 7, pp. 2717-2721, 2009.

[2] T. Zhu, C. Song, and G. Li, "Existence of mild solutions for abstract semilinear evolution equations in Banach spaces," Nonlinear Analysis. Theory, Methods \& Applications, vol. 75, no. 1, pp. 177-181, 2012.
[3] T. Cardinali and P. Rubbioni, "Impulsive mild solutions for semilinear differential inclusions with nonlocal conditions in Banach spaces," Nonlinear Analysis. Theory, Methods \& Applications, vol. 75, no. 2, pp. 871-879, 2012.

[4] X. Xue, "Nonlocal nonlinear differential equations with a measure of noncompactness in Banach spaces," Nonlinear Analysis. Theory, Methods \& Applications, vol. 70, no. 7, pp. 2593-2601, 2009.

[5] J. Liang, J. van Casteren, and T.-J. Xiao, "Nonlocal Cauchy problems for semilinear evolution equations," Nonlinear Analysis. Theory, Methods \& Applications. An International Multidisciplinary Journal, vol. 50, no. 2, Ser. A: Theory Methods, pp. 173189, 2002.

[6] J. Liang, J. Liu, and T.-J. Xiao, "Nonlocal Cauchy problems governed by compact operator families," Nonlinear Analysis. Theory, Methods \& Applications. An International Multidisciplinary Journal, vol. 57, no. 2, pp. 183-189, 2004.

[7] K. Balachandran, J. Y. Park, and M. Chandrasekaran, "Nonlocal Cauchy problem for delay integrodifferential equations of Sobolev type in Banach spaces," Applied Mathematics Letters. An International Journal of Rapid Publication, vol. 15, no. 7, pp. 845-854, 2002.

[8] Y. Lin and J. H. Liu, "Semilinear integrodifferential equations with nonlocal Cauchy problem," Nonlinear Analysis: Theory, Methods and Applications, vol. 26, no. 5, pp. 1023-1033, 1996.

[9] J. Liang and T.-J. Xiao, "Semilinear integrodifferential equations with nonlocal initial conditions," Computers \& Mathematics with Applications. An International Journal, vol. 47, no. 6-7, pp. 863-875, 2004.

[10] G. M. Mophou and G. M. N’Guérékata, "Existence of the mild solution for some fractional differential equations with nonlocal conditions," Semigroup Forum, vol. 79, no. 2, pp. 315-322, 2009.

[11] L. Byszewski and V. Lakshmikantham, "Theorem about the existence and uniqueness of a solution of a nonlocal abstract Cauchy problem in a Banach space," Applicable Analysis. An International Journal, vol. 40, no. 1, pp. 11-19, 1991.

[12] L. Byszewski, "Theorems about the existence and uniqueness of solutions of a semilinear evolution nonlocal Cauchy problem," Journal of Mathematical Analysis and Applications, vol. 162, no. 2, pp. 494-505, 1991.

[13] Y. Zhou and F. Jiao, "Nonlocal Cauchy problem for fractional evolution equations," Nonlinear Analysis. Real World Applications. An International Multidisciplinary Journal, vol. 11, no. 5, pp. 4465-4475, 2010.

[14] G. M. Mophou, "Existence and uniqueness of mild solutions to impulsive fractional differential equations," Nonlinear Analysis. Theory, Methods \& Applications. An International Multidisciplinary Journal, vol. 72, no. 3-4, pp. 1604-1615, 2010.

[15] O. K. Jaradat, A. Al-Omari, and S. Momani, "Existence of the mild solution for fractional semilinear initial value problems," Nonlinear Analysis: Theory, Methods \& Applications, vol. 69, no. 9, pp. 3153-3159, 2008.

[16] J. Wang and Y. Zhou, "Complete controllability of fractional evolution systems," Communications in Nonlinear Science and Numerical Simulation, vol. 17, no. 11, pp. 4346-4355, 2012.

[17] K. Balachandran and J. Y. Park, "Controllability of fractional integrodifferential systems in Banach spaces," Nonlinear Analysis. Hybrid Systems, vol. 3, no. 4, pp. 363-367, 2009.

[18] Y. K. Chang, J. J. Nieto, and W. S. Li, "Controllability of semilinear differential systems with nonlocal initial conditions in Banach spaces," Journal of Optimization Theory and Applications, vol. 142, no. 2, pp. 267-273, 2009. 
[19] K. Balachandran, J. P. Dauer, and P. Balasubramaniam, "Controllability of nonlinear integrodifferential systems in Banach space," Journal of Optimization Theory and Applications, vol. 84, no. 1, pp. 83-91, 1995.

[20] A. Pazy, Semigroups of Linear Operator and Applications to Partial Differential Equations, Springer, New York, NY, USA, 1983.

[21] J. Wang, M. Fečkan, and Y. Zhou, "On the new concept of solutions and existence results for impulsive fractional evolution equations," Dynamics of Partial Differential Equations, vol. 8, no. 4, pp. 345-361, 2011.

[22] J. Wang and Y. Zhou, "A class of fractional evolution equations and optimal controls," Nonlinear Analysis. Real World Applications. An International Multidisciplinary Journal, vol. 12, no. 1, pp. 262-272, 2011.

[23] Y. Zhou and F. Jiao, "Existence of mild solutions for fractional neutral evolution equations," Computers \& Mathematics with Applications, vol. 59, no. 3, pp. 1063-1077, 2010.

[24] A. Debbouche and D. Baleanu, "Controllability of fractional evolution nonlocal impulsive quasilinear delay integrodifferential systems," Computers \& Mathematics with Applications. An International Journal, vol. 62, no. 3, pp. 1442-1450, 2011.

[25] J. A. Machado, C. Ravichandran, M. Rivero, and J. J. Trujillo, "Controllability results for impulsive mixed-type functional integro-differential evolution equations with nonlocal conditions," Fixed Point Theory and Applications, vol. 2013, Article ID 66, 16 pages, 2013.

[26] H. Qin, X. Zuo, and J. Liu, "Existence and controllability results for fractional impulsive integrodifferential systems in Banach spaces," Abstract and Applied Analysis, vol. 2013, Article ID 295837, 12 pages, 2013.

[27] N. I. Mahmudov, "Approximate controllability of fractional Sobolev-type evolution equations in Banach spaces," Abstract and Applied Analysis, vol. 2013, Article ID 502839, 9 pages, 2013.

[28] Z. Liu and X. Li, "On the controllability of impulsive fractional evolution inclusions in Banach spaces," Journal of Optimization Theory and Applications, vol. 156, no. 1, pp. 167-182, 2013.

[29] A. G. Ibrahim and N. A. Al Sarori, "Mild solutions for nonlocal impulsive fractional semilinear differential inclusions with delay in Banach spaces," Applied Mathematics, vol. 4, pp. 40-56, 2013.

[30] E. Hernández and D. O’Regan, “Controllability of VolterraFredholm type systems in Banach spaces," Journal of the Franklin Institute, vol. 346, no. 2, pp. 95-101, 2009.

[31] E. Hernández, D. O’Regan, and K. Balachandran, "Comments on some recent results on controllability of abstract differential problems," Journal of Optimization Theory and Applications, vol. 159, no. 1, pp. 292-295, 2013.

[32] J. Klamka, "Constrained controllability of semilinear delayed systems," Bulletin of the Polish Academy of Sciences: Technical Sciences, vol. 49, no. 3, pp. 505-515, 2001.

[33] J. Klamka, "Constrained exact controllability of semilinear systems," Systems \& Control Letters, vol. 47, no. 2, pp. 139-147, 2002.

[34] J. Klamka, "Constrained controllability of semilinear systems with delayed controls," Bulletin of the Polish Academy of Sciences: Technical Sciences, vol. 56, no. 4, pp. 333-337, 2008.

[35] H. Qin, C. Zhang, T. Li, and Y. Chen, "Controllability of abstract fractional differential evolution equations with nonlocal conditions," Journal of Mathematics and Computer Science, vol. 17, no. 2, pp. 293-300, 2017.
[36] L. Wang, B. Yang, Y. Chen, X. Zhang, and J. Orchard, "Improving neural-network classifiers using nearest neighbor partitioning," IEEE Transactions on Neural Networks and Learning Systems, vol. PP, no. 99, pp. 1-13, 2016. 


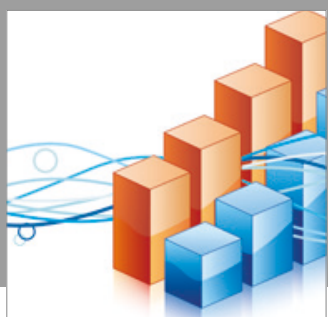

Advances in

Operations Research

vatersals

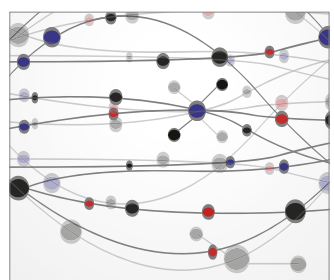

\section{The Scientific} World Journal
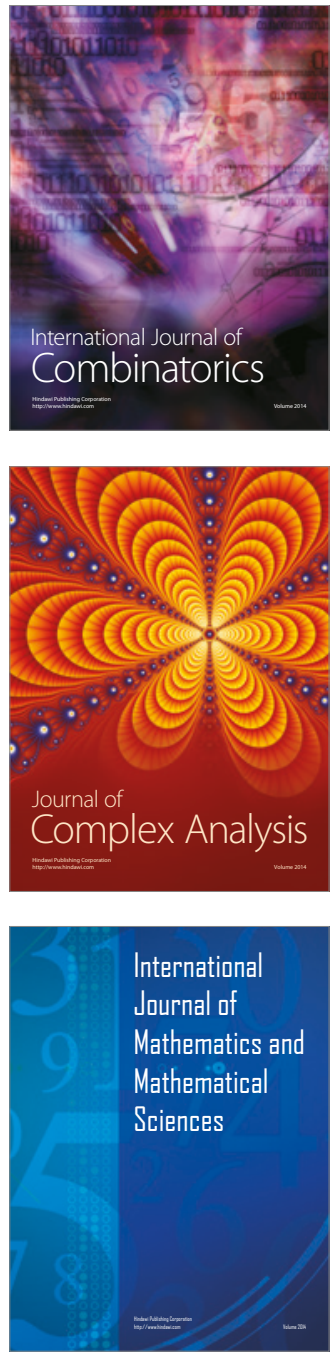
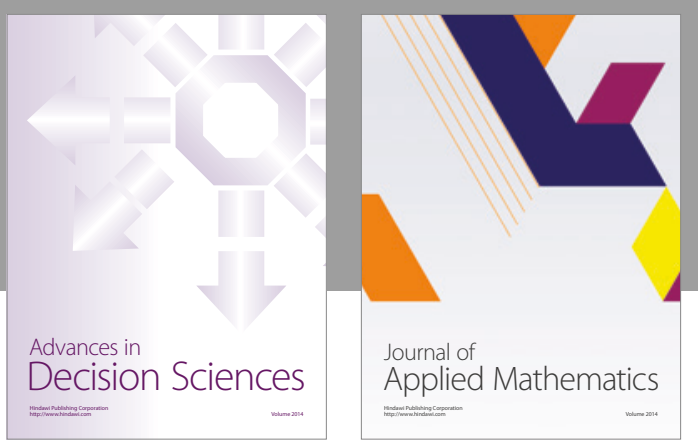

Algebra

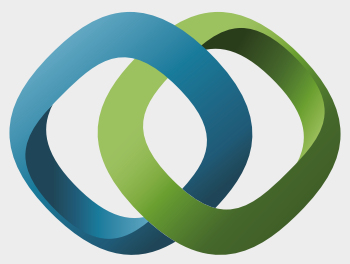

\section{Hindawi}

Submit your manuscripts at

https://www.hindawi.com
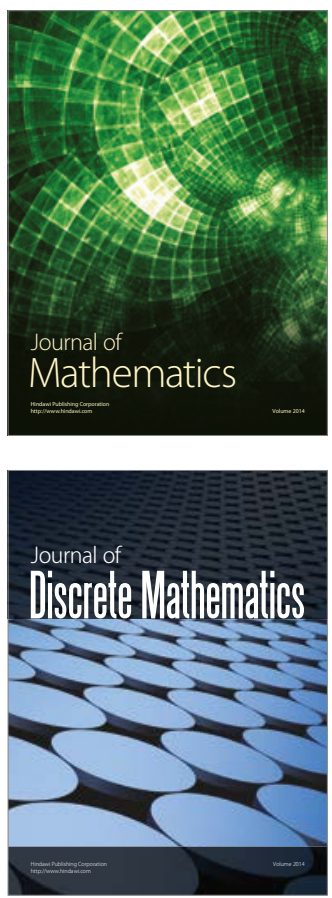

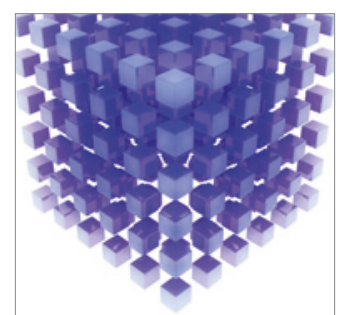

Mathematical Problems in Engineering
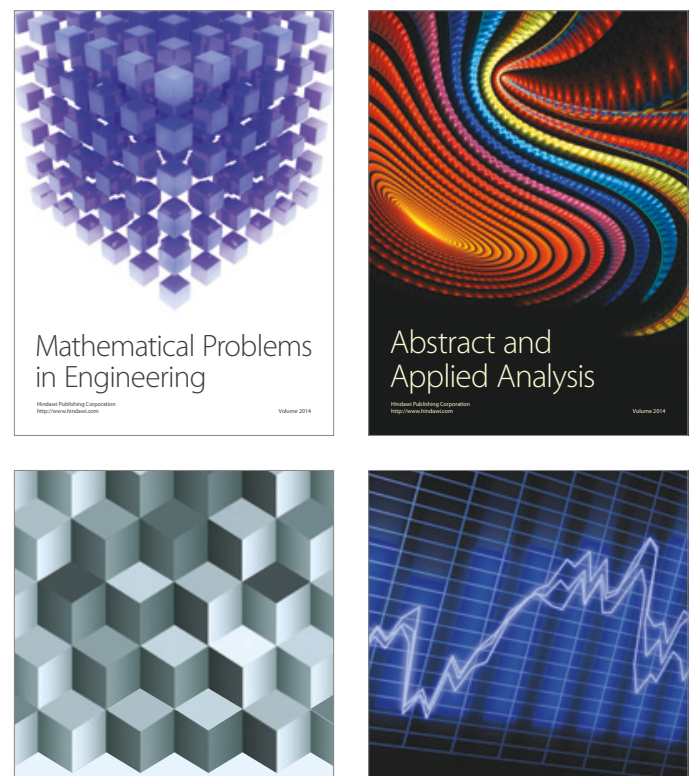

Journal of

Function Spaces

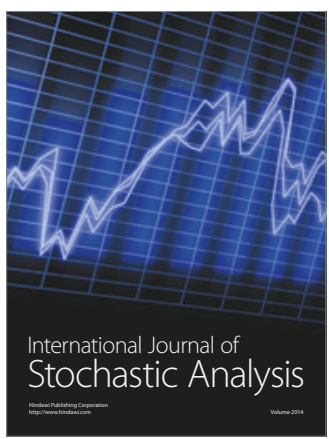

Probability and Statistics
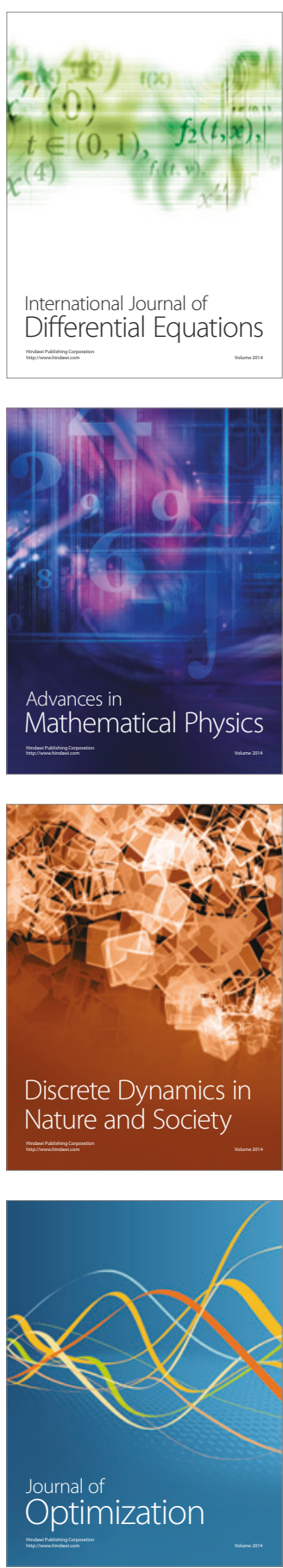